\title{
Erratum to: Diagnostic work-up of patients presenting in primary care with lower abdominal symptoms: which faecal test and triage strategy should be used?
}

\author{
Callum G. Fraser
}

\section{Erratum}

After publication of the original article [1], it came to the author's attention that there were two errors in the Conclusions section. 'Quantitative' was mistakenly written as 'qualitative' in the following part of the section:

"Quantitative estimates of $\mathrm{f}-\mathrm{Hb}$ also provide more information than qualitative $\mathrm{f}-\mathrm{Hb}$ POC tests. Simple and rapid diagnostic pathways are also advantageous. Thus, a possible strategy would be to collect a sample for quantitative measure of $\mathrm{f}-\mathrm{Hb}$ from all patients presenting with lower abdominal symptoms in primary care."

Published online: 12 October 2016

\section{Reference}

1. Fraser CG. Diagnostic work-up of patients presenting in primary care with lower abdominal symptoms: which faecal test and triage strategy should be used? BMC Med. 2016;14:139. doi:10.1186/s12916-016-0694-3. 